A N N A L E S Annales de Bretagne et des Pays de l'Ouest

Anjou. Maine. Poitou-Charente. Touraine

118-3 | 2011

La naissance de l'archéologie régionale dans l'Ouest armoricain

\title{
La naissance de l'archéologie à Nantes (1500-1860)
}

The birth of archaeology in Nantes (1500-1860)

\section{Martial Monteil}

\section{(2) OpenEdition}

Journals

Édition électronique

URL : http://journals.openedition.org/abpo/2052

DOI : 10.4000/abpo.2052

ISSN : 2108-6443

Éditeur

Presses universitaires de Rennes

Édition imprimée

Date de publication : 30 septembre 2011

Pagination : $35-53$

ISBN : 978-2-7535-1770-7

ISSN : 0399-0826

Référence électronique

Martial Monteil, «La naissance de l'archéologie à Nantes (1500-1860) 》, Annales de Bretagne et des Pays de l'Ouest [En ligne], 118-3 | 2011, mis en ligne le 30 novembre 2013, consulté le 20 avril 2019. URL : http://journals.openedition.org/abpo/2052 ; DOI : 10.4000/abpo.2052 


\title{
La naissance de l'archéologie à Nantes $(1500-1860)$
}

\author{
Jacques SANTROT \\ Conservateur en chef du patrimoine, Conseil général de Loire-Atlantique, Nantes \\ Martial MONTEIL \\ Université de Nantes, LARA, UMR 6566 CReAAH
}

\section{Les historiens de l'époque moderne et les origines antiques de Nantes}

Au XVI ${ }^{\mathrm{e}}$ siècle, si l'influence de la Renaissance et des Humanistes conduisit la société cultivée à se référer et à s'inspirer de l'Antiquité, Nantes ne pouvait se prévaloir de monuments antiques apparents, si ce n'est de quelques tronçons de la muraille romaine dissimulés sous une accumulation de constructions postérieures, et d'une partie de la modeste chapelle Saint-Étienne au milieu du cimetière de Saint-Donatien. Et encore ces deux monuments ne furent-ils rattachés à l'Antiquité tardive et au haut Moyen Âge qu'au XIX ${ }^{e}$ siècle La référence antique n'était donc pas naturelle : il a fallu des esprits éclairés pour concevoir que les traces des générations passées étaient inscrites dans le sol, pour arrêter la pioche et choisir de conserver, ou tout au moins de noter, les vestiges retrouvés à la faveur de terrassements. Il est vrai aussi que le contexte topographique particulier de la ville, parcouru par la Loire et l'Erdre et parsemé de terrains bas et humides, a fait qu'au fil des constructions médiévales et modernes les vestiges de l'époque romaine ont été enfouis à des profondeurs souvent importantes, qui n'ont été atteintes qu'avec les grands travaux mis en œuvre à la fin du XVIII ${ }^{\mathrm{e}}$ siècle et surtout au XIX ${ }^{\mathrm{e}}$ siècle Ainsi, jusque dans les années 1760, le seul monument antique signalé est-il une inscription dédicatoire aux numina des Augustes et au dieu Vulcain, découverte fortuitement en 1580 (CIL XIII, 3106, cf. infra).

En 1879, l'historien Charles Dugast-Matifeux publie une compilation de textes de ses prédécesseurs qui, complétée par d'autres sources, constitue une somme commode pour appréhender la manière dont la question des 
origines de la ville a été traitée au fil du temps ${ }^{1}$. Le premier à avoir évoqué de façon nette la création antique de Nantes est, semble-t-il, Pierre Le Baud, conseiller et aumônier d'Anne de Bretagne. Dans sa Compillation des cronicques et ystoires des Bretons, achevée vers 1480, traduite en latin par son petit-neveu Bernard d'Argentré (1582) mais publiée en 1638 seulement, il indique que "les Nantois sont ainsi nommés du nom de leur cité de Nantes, ainsi appelée dès le temps de Jules César, et est ainsi dite Nantes, pour ce qu'elle tient les nefs et qu'elle a le port convenable à les recevoir ${ }^{2}$ ". Ses successeurs vont mêler sources antiques et mythologie fabulatrice, inventant un peuple issu de la race de Noë et un roi, Namnès, qui aurait fondé la ville dans le courant du deuxième millénaire avant notre ère et serait à l'origine du nom des Namnètes. Ainsi, en 1535, Gilles Corrozet, libraire et auteur parisien, rédige un ouvrage sur les origines antiques des villes des Trois Gaules, dans lequel Nantes trouve sa place : elle aurait été fondée par « Namnès, vingt-deuxiesme roy de Gaule, fils de Galathas [qui] commença à régner un an devant que Laumédon commençast à régner à Troye, et l'an de la fondation du royaume de Gaule neuf cens et neuf, et après la fondation de Troye deux cens vingt-trois $a s^{3}$ ". Cette fable sera reprise par Bernard d'Argentré en 1582, par Charles Du Chesne en 1614, qui est l'un des premiers à ajouter que la ville a également porté le nom de Condivicnum ou Condevicnum ou Condevincum, ou encore par Jean Boutin, sieur de Chamballan et des Perrines-Boutin, en 1619, qui l'utilise sur un plan politique pour démontrer la plus grande ancienneté de Nantes par rapport à Rennes ${ }^{4}$.

Quelques décennies plus tard, en 1636-1637, le dominicain Albert le Grand, dans une rubrique consacrée à Saint-Martin de Vertou, relate la tradition qui veut que Nantes se soit étendue de part et d'autre de la Loire et ait été plus densément bâtie au sud (à l'emplacement de l'agglomération antique de Rezé/Ratiatum). Lors de la conquête romaine, la partie nord se rendit à César tandis que les occupants des quartiers sud se sauvèrent. Les soldats romains "ayant pillé cette Nantes méridionale, y mirent le feu et abbatirent ses murs, tours, portaux et édifices publics, dont on voit encore quelques vestiges au bourg qui, de cette aventure, s'appelle encore Rezay, comme qui diroit Razé. Et, depuis, Paulus Aemilius, Proconsul des Armoriques pour les Romains, voulut rebastir ce costé méridional de Nantes,

1. Dugast-MatifEuX, Charles, Nantes ancien et le pays nantais, Nantes, Morel, 1879, 583 p.

2. LE BAUD, Pierre, Histoire de Bretagne avec les chroniques des maisons de Vitré et de Laval, Paris, Gervais Alliot, 1638, p. 11.

3. Corrozet, Gilles, Les anticques erections des Gaules, Paris, Denis Janot, 1535.

4. D'Argentré, Bertrand du Plessis, L'histoire de Bretaigne, des roys, ducs, comtes et princes d'icelle : l'établissement du Royaume, mutation de ce tiltre en Duché, continué jusques au temps de Madame Anne dernière Duchesse, \& depuis Royne de France, par le mariage de laquelle passa le Duché en la maison de France, Jacques du Puys, 1588 [rédigée entre 1580 et 1582]; Du CHESNE, Charles, Les Antiquitez et recherches des villes, chasteaux..., Paris, Rocolet, 1637 (première édition en 1614), p. 1037-1038; Boutin, Jean, Apologie pour la communauté de Nantes et autres communautez de Bretagne, contre la préséance prétendue par la communauté de Rennes à la tenue des Estats de la province, Nantes, Pierre Doriou, 1619, p. 68-87. 
mais en deça la rivière de Sevre, au lieu où aboutissaient les magnifiques ponts de Nantes, lequel, encore aujourd'huy, s'appelle le bourg de Piremil, voulant dire de Paule-Aemile ${ }^{5}$ ". Dans la première moitié du XVIII ${ }^{\mathrm{e}}$ siècle, Christophe-Paul de Robien reprend à son compte cette histoire fantaisiste et ajoute que subsiste à Pirmil, au sud de la Loire et au débouché du pont principal de la ville, une tour de Pilmil, que l'on croit être un ouvrage de Paul-Émile : il s'agit en réalité d'un ouvrage du bas Moyen Âge ${ }^{6}$. Albert Le Grand, à nouveau, fixe également, après Bernard d'Argentré, les grandes lignes de l'origine du christianisme nantais dans son Catalogue chronologique et historique des evesques de Nantes : "La ville de Nantes receut la foy dès l'an soixante et dix, après la nativité [...] par la prédication de Saint Clair ${ }^{7}$."

Dès 1636, le mythe du fondateur Namnès est abandonné par DubuissonAubenay, au profit d'une lecture plus ou moins exacte des sources textuelles antiques : le peuple des Namnètes et les noms successifs de la ville, Condevicnum et Portus Namnetum sont ainsi identifiés ${ }^{8}$. Il en est de même, avec quelques modifications, par Adrien de Valois en $1675^{9}$ et par Jean Baptiste Bourguignon d'Anville en $1760^{10}$.

Mais, dans ces textes, dont la liste est loin d'être exhaustive, l'archéologie n'est pas encore présente, faute de vestiges tangibles. Il en est de même dans les premières histoires de la ville de Nantes, à l'exemple de celle de Gérard Mellier, maire de la commune, dont le manuscrit a été achevé en 1719 et ne fut publié par Léon Maître qu'en 1872. L'auteur est peu prolixe sur la période antique, se contentant de réfuter la légende de Namnès, d'attribuer à la ville le nom de Condivic et d'évoquer l'inscription dédicatoire à Vulcain $^{11}$. Il était pourtant considéré comme un antiquaire, possédait dans sa collection des monnaies antiques découvertes à Nantes et avait correspondu avec Dom Lobineau et Dom Montfaucon ${ }^{12}$. De même, l'abbé Nicolas Travers, dans le manuscrit de son Histoire des évêques, du Comté et de la

5. Le Grand, Albert, La vie, Geste, Mort et Miracles des Saints de Bretagne Armorique, Nantes, 1636-1637. Voir la réédition de ABgrall, Jean-Marie, Les vies des saints de la Bretagne armorique, Ve édition, Quimper, Peyron et Thomas, 1901, p. 528-529.

6. RoBIEn, Christophe-Paul de, Description historique, topographique et naturelle de l'ancienne Armorique, Bibliothèque municipale de Rennes, ms. 2436. Réédition de VEILLARD, Jean-Yves, Mayenne, Joseph Floch, 1974, 386 p.

7. LE GRAND, Albert, op. cit., 1636-1637. Voir la réédition de ABGRALL, Jean-Marie, Les Vies des saints de la Bretagne armorique, Ve édition, Quimper, Peyron et Thomas, 1901, p. 47* .

8. CROIX, Alain (dir.), La Bretagne d'après l'Itinéraire de monsieur Dubuisson-Aubenay, Rennes, Presses Universitaires de Rennes, Rennes, Société d'histoire et d'archéologie de Bretagne, 2006, p. 511-512.

9. ValoIs, Adrien de, Notitia Galliarum, ordine litterarum digesta..., Paris, Frédéric Léonard, 1675, p. 367-368.

10. Bourguignon D'AnVILLE, Jean Baptiste, Notice de l'ancienne Gaule tirée des monuments romains, Paris, Desaint, Saillant et Durand, 1760, p. 239-240.

11. MELLIER, Gérard, Essai sur l'histoire de la ville et du comté de Nantes, manuscrit publié par Léon Maître, Nantes, Forest et Grimaud, 1872, p. 1-5.

12. MAîTRE, Léon, dans MELLIER, Gérard, op. cit., 1872, p. X-XV. 
ville de Nantes, rédigé entre 1730 et 1750 et publié en 1836, dresse un bilan de ce qu'ont dit ses prédécesseurs de l'origine de la ville et n'évoque, au rang des documents archéologiques, que le bloc inscrit portant une dédicace à Vulcain ${ }^{13}$.

\section{Les premiers antiques de Nantes (1580-1790)}

L'évolution de l'archéologie nantaise est scandée par plusieurs étapes, mais ne s'accélère réellement qu'à compter de la fin du XVIII ${ }^{\mathrm{e}}$ siècle ${ }^{14}$. La première démarche "archéologique" semble être, à Nantes, la volonté de conserver la dédicace, aux numina des Augustes et au dieu Volianus (Vulcain), d'une plate-forme supportant une statue et de places assises, grâce à une souscription publique orchestrée par Marcus Gemellius Secundus et Caius Sedatius Florus, représentants des habitants du port (CIL XIII, $3106=$ ILS 7051, II ${ }^{\mathrm{e}}$ siècle) ${ }^{15}$. Trouvée en 1580 " dans la démolition de la porte Saint-Pierre ", au pied du rempart, cette inscription paraît être le document archéologique le plus anciennement trouvé et délibérément conservé en Loire-Atlantique. Elle fut transportée dans la cour de l'hôtel de ville à la demande de Pierre de Biré, puis, en 1623, "M. de Cornullier, chargé de la direction des bâtiments publics en qualité de Trésorier de France \& grand Voyer, fit placer ce Marbre dans la Galerie neuve construite par ses soins en 1606. Où il se voit à présent ${ }^{16}$ ". C'est bien dans la galerie basse de l'hôtel de ville qu'elle est vue par Dubuisson-Aubenay en $1636^{17}$. Comme en d'autres cités ${ }^{18}$, cette référence forte au passé marque le souci de la municipalité de

13. TRAVERS, Nicolas, Histoire civile, politique et religieuse de Nantes par l'abbé Travers, imprimé pour la première fois d'après le manuscrit original, appartenant à la bibliothèque publique de la ville de Nantes - avec des notes et éclaircissements sous la direction de SAVAGNER Auguste, I, Nantes, Forest, 1836, p. 1-12.

14. PIRAUlt, Lionel, RouAud-Rouazé, Isabelle, " Les précurseurs de l'archéologie nantaise ", 303, 50, 1996, p. 4-19; voir aussi, dans ce même volume, MonTEIL, Martial, SANTROT, Jacques, "La naissance de l'archéologie régionale dans l'Ouest armoricain : une enquête historiographique à construire " et MATHIEU, Nicolas, "L'épigraphie dans l'Ouest armoricain, historiographie et constitution des collections locales".

15. GRUTER, Jean, Inscriptiones antiquae totius orbis Romani, Heidelberg, 1603, 2 vol., p. $1074, \mathrm{n}^{\circ} 10$.

16. Moreau DE Mautour, Philippe-Bernard, Extrait de l'explication historique, d'une inscription antique conservée dans la Ville de Nantes, Nantes, 1722 (aux Archives municipales de Nantes), p. 1-23, dont p. 3-4, d'après Mémoires de Trévoux, janvier 1707, art. 13. " 1623 - Hôtel de ville. - Cette inscription romaine, découverte en 1580, a été apportée ici à la demande de $M .^{e}$ Pierre de Biré, avocat du roi au présidial, et incrusté[e] dans cette galerie par l'ordre de $M^{e}{ }^{e}$ Louis Harouys, $S^{r}$ de la Seilleraye, président à la Chambre des Comptes de Bretagne, maire de la ville" : MELLINEt, Camille, La commune et la milice de Nantes, vol. 1, Nantes, s.d. (1840), p. 276.

17. CrorX, Alain (dir.), op. cit., 2006, p. 513, n. 23, p. 514 (ill.), n. 28, et p. 531-532, où l'auteur cite Boulianus (Volianus, Vulcain), "dieu adoré par les habitants des cités d'Armorique ", et son temple de Nantes, détruit par l'évêque Eumérius "sous le consulat de Sextus Probus, au temps de l'empereur Constantin ", et p. 701-702.

18. Une vingtaine d'années plus tôt, Montaigne et De Lurbe avaient fait placer dans la maison de ville de Bordeaux, comme symbole de l'antiquité de la capitale de la Guyenne, 
manifester l'antiquité de la ville. Après quelques mentions, la relation de la découverte de 1580 ne sera publiée que près de soixante ans plus tard, en 1636, par l'oratorien Pierre Berthault ${ }^{19}$, puis en 1636-1637 par Albert le Grand $^{20}$ et par Biré de la Doucinière ${ }^{21}$. Sa traduction a fait ensuite l'objet de débats passionnés ${ }^{22}$, dont on trouve encore l'écho à date récente ${ }^{23}$ (fig. 1 et cahier couleur, fig. 2).

En 1636, Dubuisson-Aubenay traverse l'agglomération qu'il qualifie de " ville romaine " et dont il décrit avec précision, et pour la première fois, les murailles antiques ${ }^{24}$. Il analyse en outre plusieurs édifices religieux et fait même état de découvertes archéologiques ${ }^{25}$. Aux abords de la basilique Saint-Similien, il observe ainsi la " sépulture antique d'un romain chrétien [faite de] lames de plomb, [inscrite dans un caveau de briques et contenant] 7 ou 8 bouteilles de verre ${ }^{26}$ ". Il est également le premier à signaler l'ancienneté de la chapelle Saint-Étienne, située à proximité de la basilique Saint-Donatien : "Elle est très antique, faite de petite pierre quarrée, et ayant des ceintures de brique ténue, du tout à la romaine, dedans et dehors, du costé boréal. C'est sans contredit la plus ancienne muraille d'église qui soit à Nantes debout, voire l'une des plus anciennes qui soient en France ${ }^{27}$. " Par la précision de ses descriptions des monuments et par la conscience qu'il a de leur ancienneté, Dubuisson-Aubenay peut être considéré comme le premier archéologue ayant œuvré à Nantes, et plus largement dans l'Ouest

l'autel consacré à l'Auguste et au Génie de la cité des Bituriges Vivisques, daté de la fin du $\mathrm{I}^{\mathrm{er}}$ siècle et cité pour la première fois en 1534 : Bordeaux, musée d'Aquitaine, inv. 60.1.1.

19. Berthald, Petri, De ara liber singularis, Nantes, Doriou, 1636.

20. Le Grand, Albert, op. cit., 1636-137. Voir la réédition de ABGrall, Jean-Marie, op. cit., 1901, p. 48-49.

21. LA DOuCINIÈRE, Biré de, Épimasie ou relation d'Aletin le Martyr, concernant l'origine, l'antiquité, noblesse et saincteté de la Bretaigne Armorique et particulièrement des villes de Nantes et Rennes, Nantes, Doriou, 1637.

22. En 1808, P.-N. Fournier recensait 32 auteurs et études évoquant cette inscription : FourniER, Pierre-Nicolas, Antiquités de Nantes, ms 1583, Médiathèque de Nantes, fonds ancien, $\mathrm{f}^{\circ}$ 7-23. Voir, par exemple, le long article de L.-J.-M. Bizeul consacré à ce sujet : BizEul, Louis-Jacques-Marie, "Des Nannètes aux époques celtique et romaine [...]. Souschapitre IV. Antiquités romaines trouvées à Nantes ", ASANLI, XXXI, 1860, p. 237-368. Ou encore le résumé du débat qui a opposé, dans les années 1720, Philippe-Bernard Moreau de Mautour et Nicolas Travers : Dugast-MatifEuX, Charles, " Nicolas Travers, historien de Nantes et théologien ", ASANLI, XXVII, 1856, p. 254-267.

23. LE BoHEc, Yann, "L'architecture à Nantes sous le Haut-Empire romain ", dans Champeaux Jacqueline, Chassignet, Martine (dir.), Aere perennis. Hommages à Hubert Zehnacker, Paris, PUPS, 2006, p. 231-234; MALIGORNE, Yvan, "Sanctuaires et structures vicinales dans deux chefs-lieux de cités de l'ouest de la Gaule (à propos de quatre inscriptions de Nantes et Angers) ", Aremorica. Études sur l'Ouest de la Gaule romaine, 1, 2007, p. 55-71.

24. Croix, Alain (dir.), op. cit., 2006, p. 511-533.

25. Voir, pour le détail, MoNTEIL, Martial, «Les édifices des premiers temps chrétiens (IV $\mathrm{VII}^{\mathrm{e}}$ siècle) à Nantes (Loire-Atlantique) ", dans Nantes religieuse, de l'Antiquité à nos jours. Actes du colloque de l'Université de Nantes, 20-21 octobre 2006, Nantes, Société archéologique et historique de Nantes et Loire-Atlantique, Département d'Histoire de l'Art et Archéologie de l'Université de Nantes, 2008, p. 15-60.

26. CrolX, Alain (dir.), op. cit., 2006, p. 752.

27. Ibidem, p. 762. 


\section{Figure 1 - Dédicace aux numina des Augustes et à Vulcain trouvée à Nantes en 1850 (CIL XIII, 3106, If siècle) \\ (aquarelle de Fortuné Parenteau, musée Dobrée - cl. musée Dobrée)}

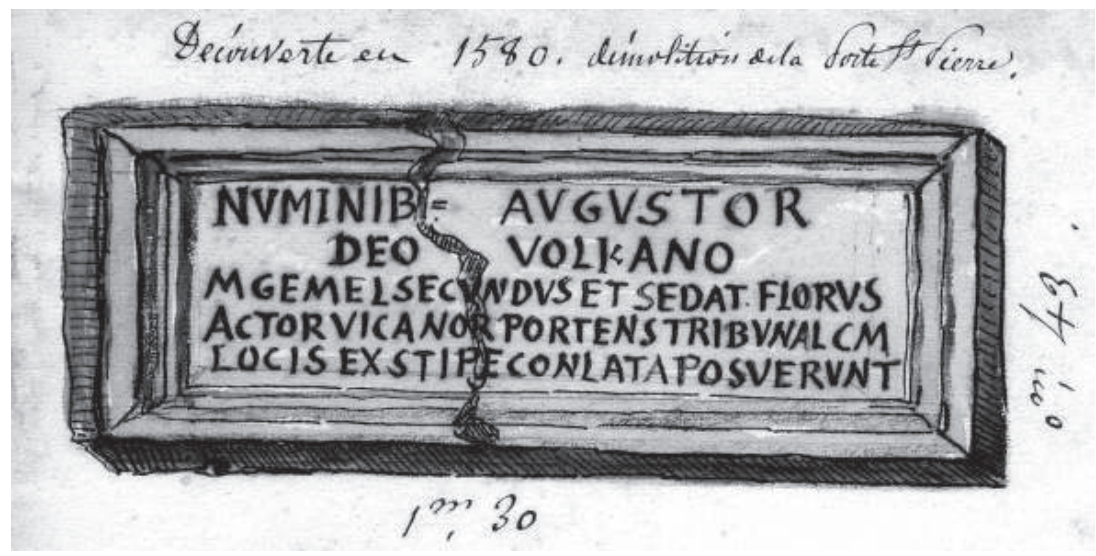

armoricain, avec une avance intellectuelle certaine sur ses successeurs des $\mathrm{XVII}^{\mathrm{e}}$ et XVIII ${ }^{\mathrm{e}}$ siècle.

Il s'écoule ensuite près d'un siècle et demi avant que quelques autres fragments d'inscriptions soient signalés à l'occasion de la destruction de portions de l'enceinte antique en 1763 puis en 1777. Grâce à l'action de l'ingénieur voyer François Cacault, les trois blocs inscrits découverts en 1763, lors de la percée de la rue de l'Évêché, sont entreposés à l'hôtel de ville. Ils y resteront jusqu'en 1790, date à laquelle ils sont remployés, malgré les protestations de son successeur, Pierre-Nicolas Fournier, dans les fondations de la colonne érigée en l'honneur de Louis XVI sur le cours Saint-Pierre où leur présence n'a pas été vérifiée récemment. D'autres blocs antiques, découverts dans le même temps, sont donnés au chirurgien Julien Minée, en dédommagement de la démolition de l'une de ses propriétés, et sont, semble-t-il, recyclés dans les nouvelles maisons qu'il fit construire rue Saint-Félix. Quant aux deux fragments de 1777, perdus, ils avaient été découverts lors de la destruction d'une portion de l'enceinte romaine comprise entre la porte Saint-Pierre et la tour du Trépied. Le texte en avait été retranscrit sur le dos d'une carte à jouer, "un six de carreau ", découvert par Louis-Jacques-Marie Bizeul ${ }^{28}$. Enfin, durant les années 17601764, qui marquent la création des cours Saint-Pierre et Saint-André à l'est

28. Découverte de 1763 : FourniER, Pierre-Nicolas, Notes sur les antiquités de Nantes, Bibliothèque municipale de Nantes, ms 1583, p. 45-48; Bizeul, Louis-Jacques-Marie, " Des Nannètes et de leur ancienne capitale ", $A S A N L I, 22,2$, 1851, p. 41-42; " Des Nannètes aux époques celtique et romaine ", BSANLI, 1859-1861, p. 276-279, p. 540. Découverte en 1777 de deux fragments perdus : Bizeul, Louis-Jacques-Marie, " Des Nannètes aux époques celtique et romaine ", BSANLI, 1859-1861, p. 349, p. 540. 
de la porte Saint-Pierre, plusieurs sépultures médiévales sont également mises au jour ${ }^{29}$. Par ailleurs, aucun document ne révèle l'existence, durant la période moderne, de cabinets de curiosités nantais semblables à celui que le marquis Christophe-Paul de Robien s'était constitué Rennes... ou, du moins, la mémoire ne s'en est-elle pas conservée.

\section{Un plus grand intérêt porté aux archives du sol (1790-1860)}

Les découvertes archéologiques à Nantes ne sont, encore aujourd'hui, que la conséquence de terrassements commandés par les travaux d'urbanisme et la mise en place d'infrastructures nouvelles. Encore faut-il de la curiosité et un esprit avisé pour identifier les vestiges, et quelque autorité pour les sauver de la pioche ou de l'engin. Il faudra attendre la fin du $\mathrm{XVIII}^{\mathrm{e}}$ siècle et le début du XIX ${ }^{\mathrm{e}}$ pour qu'un inspecteur voyer de Nantes, PierreNicolas Fournier (1747-1810), inaugure véritablement, quelque 150 ans après Dubuisson-Aubenay, la mise en œuvre d'une démarche archéologique (fig. 2). Cet ancien militaire, érudit, se retira à Nantes en 1784 et y occupa plusieurs fonctions ${ }^{30}$. Dès 1791 , il est nommé inspecteur voyer et est chargé peu après de diriger d'importants travaux d'implantation d'égouts et d'amélioration de la voirie dans le centre-ville, travaux qu'il suit jusqu'en 1808 . Curieux de nature, il prend l'initiative de noter et de dessiner les découvertes que font ses ouvriers. Dans ses notes, rédigées à la façon de procèsverbaux, P.-N. Fournier ne se borne pas seulement à une simple description factuelle, mais assortit son discours de dessins et de planches aquarellées. L'ensemble est rassemblé dans un manuscrit ${ }^{31}$, dont seule une infime partie concernant les découvertes de l'année 1808 sera éditée ${ }^{32}$, et auquel s'ajoute une synthèse datée de $1806^{33}$ ainsi qu'un plan de la ville annoté. Il est soutenu dans cette entreprise par le maire, Jean-Baptiste BertrandGeslin qui, le 8 avril 1807, ouvre un registre de 192 feuillets, "pour servir au

29. BizEul, Louis-Jacques-Marie, « Des Nannètes aux époques celtique et romaine [...]. Sous-chapitre IV. Antiquités romaines trouvées à Nantes ", BSANLI, 1859-1861, p. 549-552.

30. Il a bénéficié d'une courte nécrologie, notamment, dans Lycée Armoricain, XIII, 1829, p. 127-128.

31. FourniEr, Pierre-Nicolas, Notes sur les Antiquités de Nantes. Années 1805, 1806, 1807, Bibliothèque municipale de Nantes, ms. 1853 (1427). Ce manuscrit a fait l'objet d'un rapport d'une commission nommée par la Société des Sciences et des Arts : ATHÉNAS, Pierre-Louis, "Rapport relatif aux fouilles faites dans les années 1805, 1806 et 1807, dans la ville de Nantes, sous la direction de M. Fournier ", Procès-verbal de la séance publique de la Société des Sciences et des Arts du département de la Loire-Inférieure du 5 mai 1808, Nantes, Malassis, 1808, p. 55-87.

32. FouRNIER, Pierre-Nicolas, Second rapport sur les fouilles faites à Nantes en 1808, par ordre de M. Bertrand-Geslin... Maire de la Ville, et observées par P. Fournier, Ingénieur-civil, Inspecteur-Voyer, membre de la Société des Sciences et Arts du département de la Loire Inférieure, et de l'Académie Celtique, Nantes, imprimerie Victor Mangin, s.d. [1809], 30 p. Les pages 27 à 30 contiennent une " Note " de J.-B. Huet de Coëtlisan, secrétaire général de la Préfecture, "Sur les trois inscriptions romaines de Nantes ", datée du 20 juillet 1807.

33. FOURNIER, Pierre-Nicolas, Histoire lapidaire de Nantes, Inscriptions et monuments, 18061808, 3 vol. manuscrits, Bibliothèque municipale de Nantes, ms. 1580-1582 (1424-1426). 
secrétariat de la Mairie, à inscrire, jour par jour et à fur et à mesure qu'ils lui seront remis, d'abord les procès verbaux d'affouillements faits depuis 1797 jusqu'à l'époque présente, ensuite tous ceux de même nature et autres qui pourraient servir de preuve au Mémoire relatif à l'histoire de Nantes. [...]. Le Maire de Nantes arrête que les procès-verbaux des fouilles faites à Nantes, depuis 1797 jusqu'à 1806 seront enregistrés. Que les pierres gravées trouvées dans les fouilles seront incrustées dans la Galerie basse de l'hôtel de ville, à la suite de celle découverte en 1580. Que les objets interessans resteront déposés au Cabinet d'histoire naturelle, et charge le $S^{r}$ Fournier, Ingén ${ }^{r}$ Inspect' Voyer de l'exécution du présent, dont expédition sera remise aud ${ }^{r} S^{r}$ Fournier pour joindre aux preuves du Mémoire relatif à ces découvertes importantes pour l'histoire de Nantes ${ }^{34}$ ".

Ses notes manquent parfois de rigueur, mais ses dessins sont précis et plusieurs inscriptions et objets perdus depuis lors ne sont connus que par lui. Ses manuscrits, conservés à la médiathèque Jacques Demy de Nantes, mériteraient assurément d'être publiés ou tout au moins numérisés et diffusés ${ }^{35}$. La liste de ses interventions est longue ${ }^{36}$ et on se contentera d'en signaler l'une des plus connues, qui le conduisit à restituer un temple gréco-romain dodécastyle sur la base de la découverte, lors du creusement d'un collecteur d'égouts vers le Port-Maillard dans la rue des Petites-Écuries, de fûts de granite interprétés comme des colonnes (fig. 3). Ces derniers se sont, en réalité, avérés être des bornes milliaires, comme cela fut démontré en 1851 ( $c f$. infra).

Notons également que c'est sous l'impulsion de Pierre-Nicolas Fournier, le 14 mai 1805, qu'apparaît pour la première fois à Nantes, la notion de périmètre archéologique et de secteur sauvegardé : "Nous pensons qu'il est intéressant pour l'histoire, que l'on place dans ce lieu [sous la porte SaintPierre] une inscription invitative de ne permettre aucuns travaux dans cette partie de la Ville, qui a été la $1^{\text {re }}$ habitée, pour que les administrations en soient prévenues, car l'Évêché peut être considéré comme le point central d'une grande circonférence sous le sol duquel se trouve ensevelie tout ce que les anciens nous ont laissé de plus remarquable à Nantes ${ }^{37}$. "Les travaux de P.-N. Fournier s'inscrivent dans un contexte qui voit la création, en 1805, de l'Académie celtique, fondée sous les auspices de Napoléon ${ }^{\mathrm{er}}$, dont il fut membre et qui se propose "de recueillir de nouvelles connaissances sur les antiquités de la Gaule avant qu'elle ne devienne romaine".

En parallèle, entre cette fin du XVIII ${ }^{\mathrm{e}}$ siècle et les premières décennies du XIXe, quelques autres Nantais se singularisent par l'intérêt qu'ils por-

34. FOURNIER, Pierre-Nicolas, Fouilles et découvertes de médailles, Inscriptions anciennes

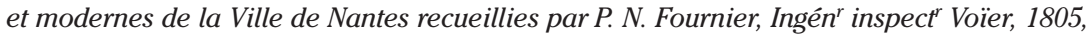
$A N$ XIII RF, 1807, $\mathrm{f}^{\circ} 1$, Archives municipales de Nantes, $\mathrm{n}^{\circ} 426$.

35. Un tel vœu a été émis dès 1808: "inviter M. le Maire à le faire imprimer et graver, aux frais de la Mairie ", ATHÉNAS, Pierre-Louis, op. cit., 1808, p. 87.

36. Voir, dans ce même volume, ANWAR, Nasrine et SANTROT, Jacques, "Principales découvertes archéologiques à Nantes et en Loire-Inférieure jusqu'en 1943 ».

37. FourniER, Pierre-Nicolas, op. cit., 1807, $\mathrm{f}^{\circ} 5, \mathrm{n}^{\circ} 15$, Archives municipales de Nantes, $\mathrm{n}^{\circ} 426$. 
Figure 2 - Portrait de PierreNicolas Fournier (1747-1810) placé en tête de ses Notes sur les Antiquités de Nantes (cl. Bibliothèque municipale de Nantes)

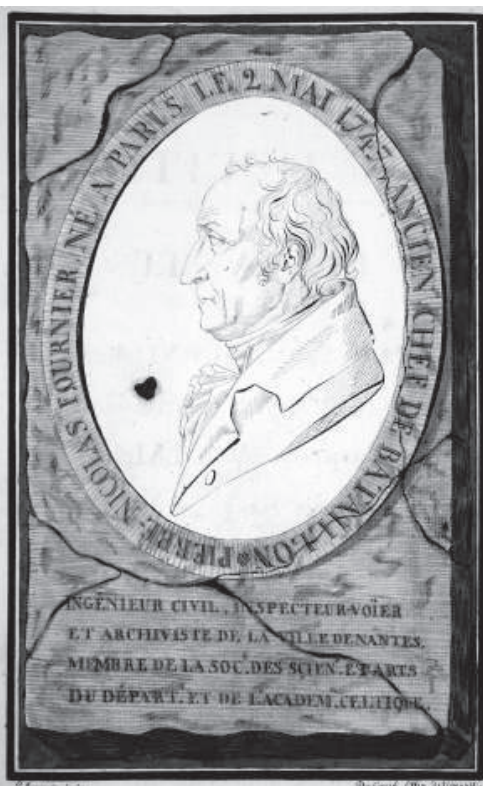

tent à l'archéologie locale, même si leur production scientifique n'a pas l'ampleur de celle de P.-N. Fournier. Il en est ainsi de Pierre-Louis Athénas (1752-1829), directeur de la Monnaie de Nantes à compter de 1795 (fig. 4), de Charles-Marie Richard, député de la Loire-Inférieure, de Jacques-Jean Le Cadre (1767-1846), entrepreneur de commerce et homme de lettres. On retrouve ces trois hommes dans la description d'objets découverts, en 1827, lors de travaux d'approfondissement de l'Erdre, en lien avec la construction du canal de Nantes à Brest, à l'embouchure de la rivière dans la Loire, au bas de la rue de la Boucherie. La publication comprend notamment trois chenets en terre cuite à tête de bélier, une statuette en bronze représentant Bacchus (fig. 5),

Figure 3 - Restitution de la colonnade $\mathrm{du}$ " temple carré » de la rue du Port-Maillard (FOURNIER, PierreNicolas, Notes sur les Antiquités de Nantes..., ms. 1583 - cl. Bibliothèque municipale de Nantes)

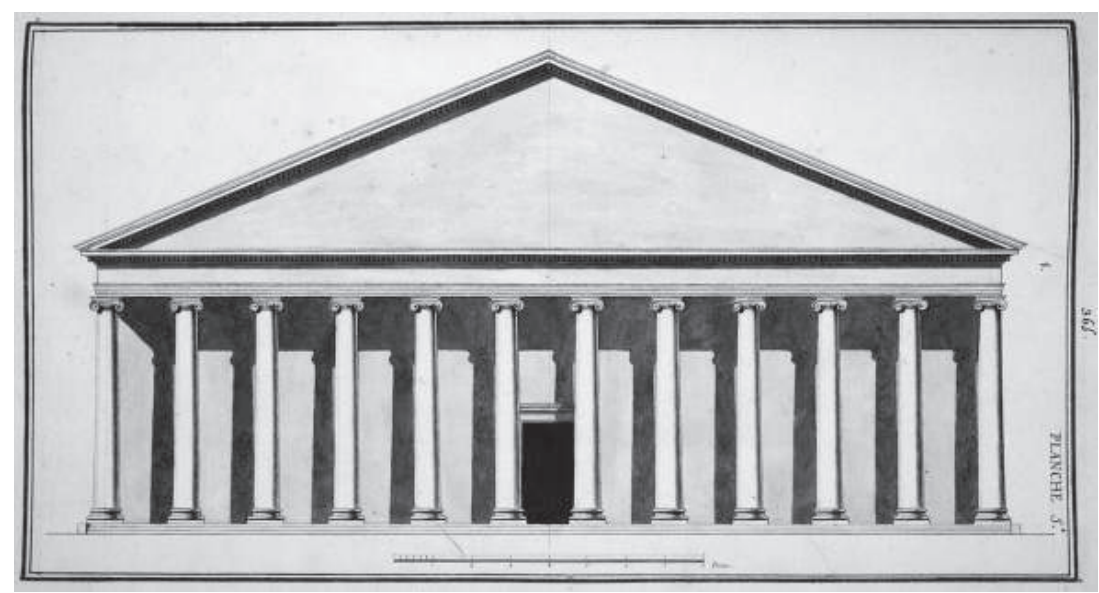


propriété du député Richard, des monnaies ou encore un bas-relief en terre cuite $^{38}$. Mais les conditions d'observation sont bien différentes de celles qui ont présidé aux découvertes de P.-N. Fournier : "Il a été également extrait du même endroit des médailles en or et en argent qui ont disparu dans les creusets de l'orfêvre [...]. D'autres ont été préservées de cette fatale destruction, mais, je ne les ai pas vues [...] il se passe peu de jours qu'il ne soit découvert de nouveaux objets dans les fouilles du canal; mais il existe maintenant une active concurrence entre les amateurs et entre les marchands, pour se procurer à l'envie les découvertes quotidiennes ${ }^{39}$."

\section{Figure 4 - Portrait de Pierre- Louis Athénas (1752-1829)}

(musée Dobrée, inv. 850.23.1 cl. musée Dobrée)

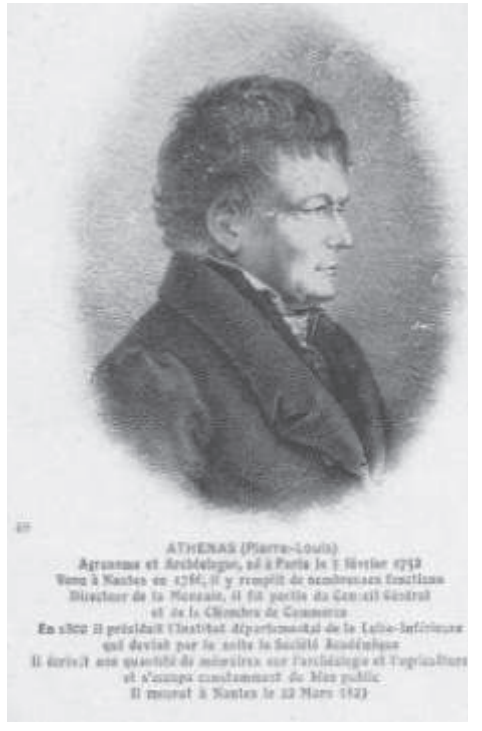

Figure 5 - Dessin d'une statuette en bronze de Bacchus, découverte dans le lit de l'Erdre au niveau de la rue de la Boucherie, en 1827

(Propriété du député Richard, elle a disparu. Litho. de Mellinet dans Lycée Armoricain, X, 1827 - cl. musée Dobrée)

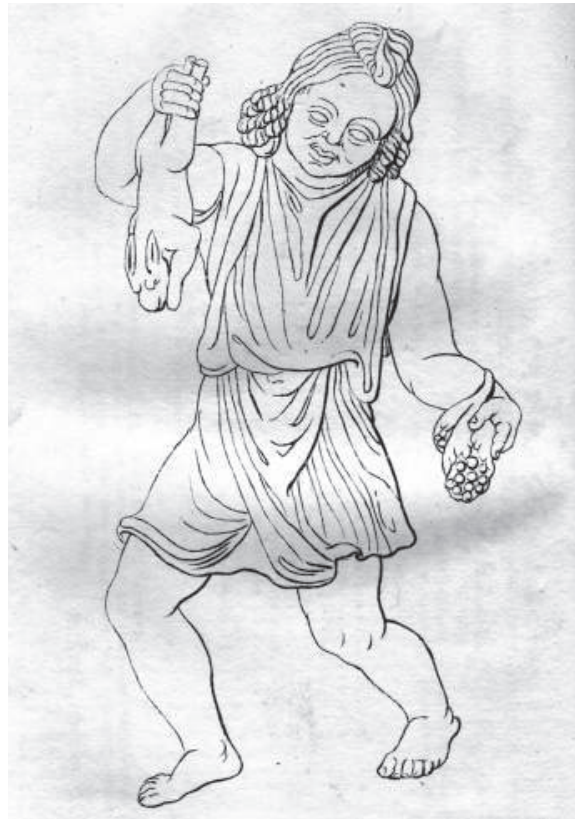

38. AthÉNAS, Pierre-Louis, "Antiquités trouvées à Nantes ", Lycée Armoricain, X, 1827, p. 244-245; idem, "Idole du sommeil trouvée à l'entrée du canal de Bretagne, Lycée Armoricain, X, 1827, p. 407; LE CADRE, Jacques-Jean, "Communication à la Société académique du département de la Loire-Inférieure de quelques observations sur des antiquités découvertes ", Lycée Armoricain, X, 1827, p. 278-293; id., "Addition aux antiquités découvertes à Nantes ", Lycée Armoricain, X, 1827, p. 295-296.

39. LE CADRE, Jacques-Jean, op. cit, 1827, p. 287-288, p. 295. Voir aussi Bizeul, LouisJacques-Marie, " Des Nannètes aux époques celtique et romaine ", BSANLI, 1862, p. 77-95. 
Certaines de ces découvertes, effectuées entre les années 1790 et 1830, vont enrichir le futur musée d'archéologie, mais beaucoup ont disparu. L.-J.-M. Bizeul note ainsi qu'“ on ne sait ce qu'est devenu, à la mort de M. Richard, le cabinet d'antiquités choisies qu'il s'était plu à former ${ }^{40}$ " ou encore le fait que les monnaies déposées par P.-N. Fournier à la Bibliothèque municipale sont perdues ${ }^{41}$. Une commission de la Société Archéologique, créée en 1850 (cf. infra), "exprime son regret bien senti de la disparition d'une foule d'objets appartenant à l'époque romaine, trouvés dans les fouilles faites à Nantes en 1805, 1806, 1807, déposés, dans le temps, à la bibliothèque municipale et au Muséum d'histoire naturelle. Les objets disparus, indiqués ou décrits par Fournier, consistaient en sculptures, ustensiles, briques, tuiles, poteries, meules en basalte, monnaies et médailles en or, en argent, en bronze ${ }^{42}$ ". Léon Maître vient confirmer plus tard ces pertes et d'autres : "La plupart de nos richesses archéologiques ont disparu. Qu'est devenue la collection de médailles de Fournier? Qu'est devenu le cabinet de Richard jeune? Celui d'Athénas ${ }^{43}$ ?"

Les données extraites du sous-sol ne suffisent toutefois pas à élaborer une véritable synthèse sur les origines antiques de la ville et leur diffusion en dehors du cadre local reste peu importante. En 1817, Claude-Madeleine Grivaud de la Vincelle publie toutefois quatorze inscriptions et monuments de Nantes ${ }^{44}$, mais les quatre planches qui les représentent ne seront pas éditées ${ }^{45}$. En 1825, Jean-François Le Boyer, dans une histoire des communes du département de la Loire-Inférieure, ne signale que trois inscriptions conservées à l'hôtel de ville et expédie en deux pages l'histoire antique de Nantes ${ }^{46}$. Il faut attendre 1839 pour qu'Ange Guépin, dans son Histoire de Nantes, dresse, en se fondant sur les travaux de Pierre-Nicolas Fournier, une première esquisse de la topographie urbaine de la ville antique. Pour l'époque gauloise, il restitue, sans preuves mais en ayant conscience des modifications du cadre naturel, une " pauvre bourgade habitée par une race forte et guerrière ». Pour l'époque romaine, il note : "nous savons [...] surtout

40. Bizeul, Louis-Jacques-Marie, " Des Nannètes aux époques celtique et romaine ", BSANLI, 1859-1861, p. 84.

41. Bizeul, Louis-Jacques-Marie, "Des Nannètes aux époques celtique et romaine ", BSANLI, 1862, p. 541, 557.

42. VANDIER, Jules-Théodore-Armand, "Rapport sur les antiquités de Nantes ", BSANLI, 1859-1861, p. 80.

43. MAîTRE, Léon, Géographie historique et descriptive de la Loire-Inférieure, I, Les villes disparues des Namnètes, Nantes, Émile Grimaud, 1893, p. 395.

44. GRIVAUd DE LA VinCELLE, Claude-Madeleine, Recueil des monumens antiques, la plupart inédits et découverts dans l'Ancienne Gaule. Ouvrage enrichi de cartes et planches en taille-douce, qui peut faire suite aux recueils du Comte de Caylus et de Félix Le Royer de La Sauvagère; dédié à son altesse royale, monseigneur le prince héréditaire de Bavière, Treuttel et Wurtz, 1817.

45. Elles seront publiées dans le Bulletin de la Société Archéologique de Nantes et de la Loire-Inférieure en 1862, à la suite de la p. 99.

46. Le BOYER, Jean-François, Notices sur les villes et les principales communes du département de la Loire Inférieure et en particulier sur la ville de Nantes, Nantes, Forest, seconde édition, 1825, p. 25-28, p. 45-46. 
par les fouilles de l'architecte Fournier, que notre ville a eu un grand nombre d'habitations romaines sur la pointe de l'angle formé autrefois par le Seil et l'Erdre". Il évoque les inscriptions qui montrent que "Nantes possédait une bourse et un tribunal de commerce ", le temple restitué par P.-N. Fournier dans la rue du Port-Maillard et plusieurs autres découvertes, mais paraît ignorer l'existence d'une enceinte de l'Antiquité tardive ${ }^{47}$.

La première véritable synthèse de l'ensemble des données est l'œuvre de Jacques-Louis-Marie Bizeul (1785-1861), grand archéologue amateur et initiateur de l'étude des voies romaines de l'Ouest armoricain (fig. 6). Il a produit, au sein d'une ample bibliographie, une étude complète du territoire des Namnètes en y incluant l'ensemble des données disponibles sur Nantes. Bien qu'il ait dénié à la ville le titre de chef-lieu de cité avant le IV ${ }^{\mathrm{e}}$ siècle, privilégiant l'agglomération de Blain dont il était originaire, il en propose un panorama complet et rigoureux en dressant le premier inventaire complet des vestiges connus et en décrivant de façon détaillée le circuit de l'enceinte tardive ${ }^{48}$.

Dans les années 1827-1860, quelques autres découvertes ponctuelles sont signalées ${ }^{49}$, mais les observations les plus importantes ont lieu en 1847-1848 et en 1851. Celles de 1847-1848 sont liées à la destruction du château du Bouffay, jusque-là palais de justice et prison. Sa démolition a

47. GuÉPIN, Ange, Histoire de Nantes, Nantes, Mellinet, 1839, p. 5-9.

48. Sa synthèse sur les Namnètes a été découpée en plusieurs articles : Bizeul, LouisJacques-Marie, « Des Nannètes et de leur ancienne capitale », ASANLI, 22, 2, 1851, p. 41-42; " Des Nannètes aux époques celtique et romaine. $1^{\text {re }}$ partie (époque celtique) ", $R P O$, I, 1853 , p. $363-371$, p. $391-407$, p. $435-442$; RPO, II, 1854, p. $96-109$, p. 278-288, p. 714-725, p. 813-834; RPO, III, 1855 , p. 72-82, p. 667-673, p. 728-742; « Des Nannètes aux époques celtique et romaine. $2^{\text {e }}$ partie (époque romaine) ", BSANLI, I, 1859-1861, p. 114-134, p. 151169 , p. 209-247, p. 276-313, p. 335-370, p. 538-562; BSANLI, II, 1862, p. 77-99.

49. Liste des principales découvertes enregistrées entre 1827 et 1860 , à compléter par BIZEuL, Louis-Jacques-Marie, « Des Nannètes aux époques celtique et romaine [...]. Souschapitre IV, Antiquités romaines trouvées à Nantes ", BSANLI, II, 1862, p. 77-99.

- 1830 - Près de l'église Saint-Similien, découverte par Jean-François Le Boyer d'un égout ou d'un canal, antique ou plus récent : LE BOYER, Jean-François, « Rapport [...] sur un souterrain découvert à Saint-Similien ", ASANLI, I, 1830, p. 163-172.

- 1834 - Place et rue Travers, près de l'église Saint-Saturnin, M. Mareschal a observé, dans les fondations de l'enceinte, des fragments d'architecture et des canalisations en granite : " Séance du 5 août 1848 ", BSANLI, I, 1859-1861, p. 189-190.

- 1836 - La démolition de la tour du Trépied, rue du roi Albert, permet de recueillir, dans les fondations, des fragments d'architecture, de deux sphinges, d'une tête en grès roux ainsi que des céramiques : BizEuL, Louis-Jacques-Marie, op. cit., 1862, p. 95-96.

- 1838 - Dans le transept nord de la cathédrale, découverte par l'architecte Seheult de deux inscriptions, dont l'une dédiée à Mars Mullo, ainsi que d'une colonne et d'un chapiteau en marbre blanc: VERGER, François, MSNAF, 1840, p. XXXIV-xxxv; BIZEUL, LouisJacques-Marie, op. cit., 1862, p. 96-97.

- 1849 - Dans le chœur de la cathédrale, découverte d'une déesse de l'abondance assise : MAîTRE, Léon, Géographie historique et descriptive de la Loire-Inférieure, I, Les villes disparues des Namnètes, Nantes, Émile Grimaud, 1893, p. 412.

- 1858 - Sous le clocher de l'église Sainte-Croix, découverte par l'architecte Henri Driollet d'une salle voûtée sur hypocauste : «Séance du 7 juin 1859 », BSANLI, 1859-1861, I, p. 35; plan et description produits par MAîTRE, Léon, op. cit., 1893, p. 386, 407-408. 
Figure 6 - LouisJacques-Marie Bizeul (1785-1861)

(BMN-d'après 303,

$50,1996, p .14-$ cl. B. Renoux)

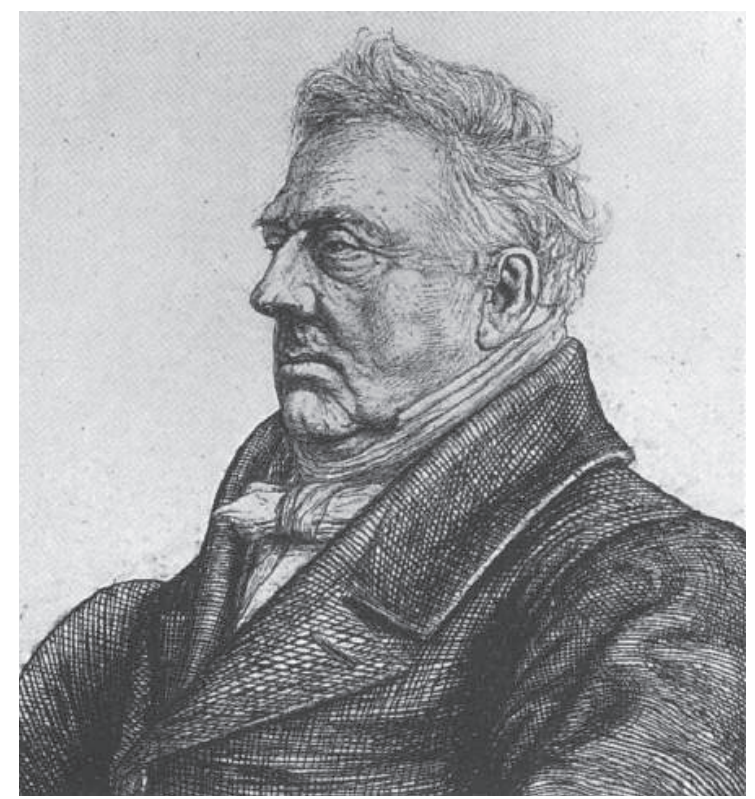

été surveillée, à la demande du préfet, par une commission de la Société archéologique, alors fraîchement créée. Outre des observations faites sur une tour de l'enceinte antique et sur une longueur de 60 mètres de courtine, on y recueillit plusieurs blocs sculptés représentant une scène d'amazonomachie qui décorait vraisemblablement un mausolée ${ }^{50}$.

En octobre 1850, le maire de Nantes, Évariste Colombel, invite la Société archéologique, fondée cinq ans auparavant, "à rechercher les objets d'antiquité qui peuvent exister dans la ville, afin de les sauver de la destruction, et les faire servir d'éléments à une histoire locale dont l'absence se fait sentir". À la suite de cette initiative louable, dont bien des maires actuels devraient s'inspirer, une commission de six membres est alors élue : présidée par Louis-Jacques-Marie Bizeul, elle comprend le président de la Société archéologique, Théodore Nau, architecte de l'achèvement de la cathédrale, l'adjoint au maire Frédéric Huette, l'abbé Rousteau, professeur d'archéologie au Petit Séminaire, le baron Olivier de Wismes et l'archiviste départemental Jules-

- 1858 - Découverte fortuite d'une inscription dans les fondations de l'enceinte, rue Saint-Jean : LEGENDRE, Alfred-Mathurin-Gustave, " Nantes à l'époque gallo-romaine, d'après les découvertes faites à la porte Saint-Pierre ", $A S A N L I, 7^{\mathrm{e}}$ série, 2, 1891, p. 122-123.

- 1859 - Dans la cathédrale, le mur de l'enceinte antique est observé : " Séance du 5 avril 1859 », BSANLI, 1859-1861, I, p. 34.

- 1860 - Rue des Pénitentes, fouille de l'enceinte antique et prélèvement de blocs en remploi dans sa fondation : "Séance du 6 mars 1860 ", BSANLI, I, 1859-1861, p. 174.

50. VANDIER, Jules-Théodore-Armand, " Antiquité romaines de Nantes... ", BAAB, 1852, 4, p. 17-20. 
Théodore-Armand Vandier. Lors de la séance du 3 décembre 1850, cette commission lit son rapport, dans lequel elle propose un vaste programme de sept fouilles et le relevé d'un certain nombre d'éléments d'époque médiévale et moderne. Elle précise que « dans l'esprit de la Commission, les recherches projetées doivent avoir un autre but que la découverte d'inscriptions, de colonnes, de statues, de sculptures, de monnaies, de médailles, d'ustensiles, de poteries, d'armes, de bijoux; elles doivent aider à préciser l'étendue et la direction du périmètre de la cité romaine de Nantes ${ }^{51} "$.

En 1851, la première fouille programmée réalisée à Nantes est ainsi mise en œuvre, rue du Port-Maillard (actuelle rue des Petites-Écuries), sous la conduite de l'architecte Henri Driollet et avec l'aide d'une subvention municipale. C'est là, qu'en 1805, Pierre-Nicolas Fournier avait observé des colonnes sur la base desquelles il avait restitué un vaste temple. Malgré de très mauvaises conditions climatiques, les fouilles de 1851 vont permettre, en réalité, d'identifier plusieurs bornes milliaires, recyclées dans les fondations de l'enceinte tardive ${ }^{52}$.

En juin 1856, enfin, la communauté archéologique nantaise est désormais suffisamment mûre pour accueillir la XXIII ${ }^{\mathrm{e}}$ session du congrès de la Société Française d'Archéologie ${ }^{53}$.

\section{Les sociétés savantes nantaises et l'archéologie (1798-1860)}

Les archéologues, érudits et collectionneurs de la fin du XVIII ${ }^{\mathrm{e}}$ siècle contribuent également à la fondation, en 1798 seulement ${ }^{54}$, de l'Institut départemental des Sciences et des Arts de la Loire-Inférieure, première société savante qui intègre dans ses préoccupations, de façon toutefois accessoire, la connaissance du passé. En 1802, cet Institut départemental se transforme en Société des Sciences et des Arts du département de la Loire-Inférieure ${ }^{55}$ et, après une brève interdiction politique, devient, en 1817, la Société Académique dont les Annales sont publiées à compter de 1830. Membre de cette société, l'armateur et négociant Thomas (I) Dobrée (1781-1828), le père du mécène, arpente le littoral vers 1819-1823 pour peindre à l'aquarelle des portraits de "monuments druidiques " (mégalithes) ou des plates tombes médiévales; il visite, peint et décrit les antiques de Nîmes et les mégalithes de Guernesey, et collectionne les monnaies de Corseul et du Pays de Retz ${ }^{56}$.

51. VANDIER, Jules-Théodore-Armand, "Rapport sur les antiquité de Nantes ", BSANLI, 1859-1861, I, p. 72-88; idem, "Antiquité romaines de Nantes... ", BAAB, 1852, 4, p. 20-26.

52. VANDIER, Jules-Théodore-Armand, "Antiquité romaines de Nantes... ", BAAB, 1852, 4, p. $26-28$.

53. CAF. Séances générales tenues à Nantes, en 1856, à Verneuil, au Neubourg et à Louviers par la Société Française d'Archéologie pour la conservation des Monuments Historiques, Paris, Derache, Caen, Hardel, 1857, 395 p.

54. $1^{\text {er }}$ fructidor An VI-18 août 1798.

55. 25 floréal An X-15 mai 1802.

56. Santrot, Jacques, "Un certain intérêt pour l'archéologie ", dans Aptel, Claire, Biotteau, Nathalie, Richard, Marie, SAnTrot, Jacques, Thomas Dobrée, un homme, un musée, cat. d'expo., Nantes et Paris, musée Dobrée et Somogy, 1997, p. 74-81. 
Plusieurs membres éminents de la Société Académique seront ensuite les fondateurs ou les animateurs de la Société archéologique de Nantes et de Loire-Inférieure, comme Louis-Jacques-Marie Bizeul, Léon Maître, qui en sera président en 1882, l'ingénieur René de Kerviler, qui invente le "chronomètre préhistorique de Saint-Nazaire ${ }^{57}$ " ou encore l'historien républicain Charles Dugast-Matifeux, qui apporte à Michelet, en exil à Nantes, la documentation sur les Guerres de Vendée nécessaire à son Histoire de la Révolution, et qui fut l'exécuteur testamentaire du juriste et archéologue vendéen Benjamin Fillon dont il recueillit une partie des collections.

En 1843 est organisé à Vannes, le premier congrès de l'Association Bretonne, à vocation d'abord agricole, mais qui se dote, en 1844, au congrès de Rennes, d'une section "archéologie", sur la suggestion d'Arcisse de Caumont. Cette "classe d'archéologie" connaît un tel succès qu'elle se subdivise en sociétés archéologiques départementales ${ }^{58}$. Ainsi, le 9 août 1845, est créée la Société archéologique et historique de Nantes et de la Loire-Inférieure à l'occasion du congrès de l'Association Bretonne qui se tient à Nantes : elle comprend 40 membres et est placée sous la présidence de Théodore $\mathrm{Nau}^{59}$.

Cette Société, qui bénéficie de subventions du Conseil général dès 1849, et de la Ville de Nantes à partir de 1850, est constituée de trois sections : "histoire et géographie", archéologie monumentale et numismatique, inscriptions et archives. Elle publie son premier bulletin annuel en 1859 et connaît un pic d'effectifs vers $1860^{60}$, une décrue dans les années 1860 et, surtout, vers 1870 (guerre franco-prussienne), une reprise puis une stagnation vers 1875-1895. Son apogée se situe vers 1885-1900, période où elle compte 135 membres ${ }^{61}$. Jusque vers 1870 , les membres de la Société sont d'abord architectes, médecins, artistes et avocats, et, dans une moindre mesure, ecclésiastiques, fonctionnaires et commerçants. Ils sont plutôt jeunes : $40 \%$ ont moins de 45 ans. Les femmes sont longtemps absentes de la Société : "La Société Archéologique est une société d'élite. Elle se distingue par la nature sérieuse de ses études, par le choix et le caractère de ses membres, tous recrutés parmi ces hommes honorables qui, dédaignant la vie frivole, cherchent leurs délassements dans les jouissances élevées de l'esprit ${ }^{62}$."

57. SANTROT, Jacques, « Le chronomètre préhistorique de Saint-Nazaire ", Place publique, 02, mars 2007, p. 64-65.

58. La Société Bretonne est dissoute en 1859, ses membres, républicains, ayant refusé d'accueillir Napoléon III lors de sa visite en Bretagne.

59. Association bretonne. Classe d'archéologie. Congrès tenu à Nantes en 1845. ProcèsVerbaux, Rennes, Jasions, 1846, p. 109. Voir aussi : VANDIER, Jules-Théodore-Armand, " Notice sur la Société Archéologique de Nantes ", BSANLI, I, 1859-1861, p. 25-30.

60 . Au $1^{\text {er }}$ juillet 1859 , elle compte 118 membres titulaires et 18 membres honoraires : BSANLI, I, 1859-1861, p. 2-7.

61. DuRAND, Franck, La Société archéologique de Nantes et de Loire Inférieure de 1845 à 1920, Nantes, Université de Nantes, mémoire de maîtrise d'histoire, 1990, 135 p., ill.

62. CAHOUR, abbé Abel, « Procès-verbal du 15 décembre 1868 », BSANLI, 8, 1868, p. 253. Ce n'est qu'en 1895 que, grâce à Pitre de Lisle du Dreneuc, il sera permis aux femmes 


\section{La naissance des collections d'archéologie à Nantes (1849) et la création du musée départemental d'Archéologie (1860)}

Dès 1849, les membres de la Société archéologique de Nantes et de la Loire-Inférieure commencent à collecter les vestiges archéologiques et les exposent ou les rangent dans une salle mise à disposition par la ville, dans l'ancienne école de dessin de la rue du Moulin, locaux qui, ouverts au public le $1^{\mathrm{er}}$ mai 1849 , se révèlent vite exigus. En 1856, le préfet, avec l'assentiment du Conseil général, met à disposition de la Société la chapelle de l'Oratoire, local municipal (fig. 7). Cet événement consacre de fait l'ouverture officielle d'un musée Archéologique à Nantes, tenu par les membres de la Société archéologique et qui bénéficie d'un premier catalogue de ses collections $^{63}$ : il est inauguré en juin de la même année, à l'occasion du congrès de la Société Française d'Archéologie ${ }^{64}$. Les collections y seront pourtant, à nouveau, très rapidement à l'étroit, d'autant que les sacristies, aux surfaces très modestes, sont insuffisantes pour accueillir des réserves. Le conservateur d'alors, Pitre de Lisle du Dreneuc, demande et obtient de la Société archéologique l'autorisation d'effectuer un "tri sélectif" dans les tiroirs servant de réserve archéologique. Passionné de Préhistoire, il a la main plus lourde dans le tri des collections gallo-romaines.

La gestion d'un musée apparaît désormais trop complexe et onéreuse aux membres de la Société qui décident de confier au Département la conservation de leurs collections et la mission pédagogique d'en faire un musée. L'acceptation de ce don, en 1860, consacre la création du musée départemental d'Archéologie, qui reste toutefois hébergé dans la chapelle, municipale, de l'Oratoire (1856-1896). 1040 objets ou ensembles sont alors inscrits dans la liste de ce don et au catalogue de l'exposition. Après la mort de Thomas Dobrée (1895) et ses dons et legs du palais Dobrée, du manoir de la Touche et de ses collections, le musée départemental d'Archéologie sera transféré par le Département sur le site du musée Dobrée : déménagement des collections de l'Oratoire en 1897 et, le 8 janvier 189965, ouverture au public des collections d'archéologie au rez-de-chaussée du palais Dobrée, avant leur installation dans le manoir de la Touche en 1911.

Après 1860, l'archéologie nantaise poursuivra son évolution, scandée par des moments d'intense activité et de long silence ${ }^{66}$. Il faudra toutefois

d'être associées correspondantes, ne participant aux réunions et excursions que sur invitation... Les deux premières femmes, des aristocrates, ne seront admises qu'en 1900.

63. GuÉRAUD, André et PARENTEAU, Fortuné, Catalogue et description des objets d'art du musée archéologique de Nantes et de la Loire-Inférieure, Nantes, Guéraud et Cie, 1856, 108 p.

64. VANDIER, Jules-Théodore-Armand, op. cit., BSANLI, I, 1859-1861, p. 29-30.

65. SANTROT, Jacques et MouthenOt, Anne, " Première muséographie ", dans APTEL, Claire, Biotteau, Nathalie, Richard, Marie, SANTROT, Jacques, Thomas Dobrée, un homme, un musée, op. cit., p. 303-307.

66. On se reportera notamment, pour ne citer que quelques références parmi les plus récentes, à : PIRAULT, Lionel, RouAUD-RouAZÉ, Isabelle, "Les précurseurs de l'archéologie 
Figure 7 - Musée de

Nantes, “ Antiquités romaines ", musée départemental d'Archéologie dans la chapelle de l'Oratoire (extrait de MAîTRE, Léon, Géographie historique et descriptive de la LoireInférieure, $I$, Les Villes disparues des Namnètes, 1893, t. 1, éd. Emile Grimaud, entre les p. 370 et 371$)$.

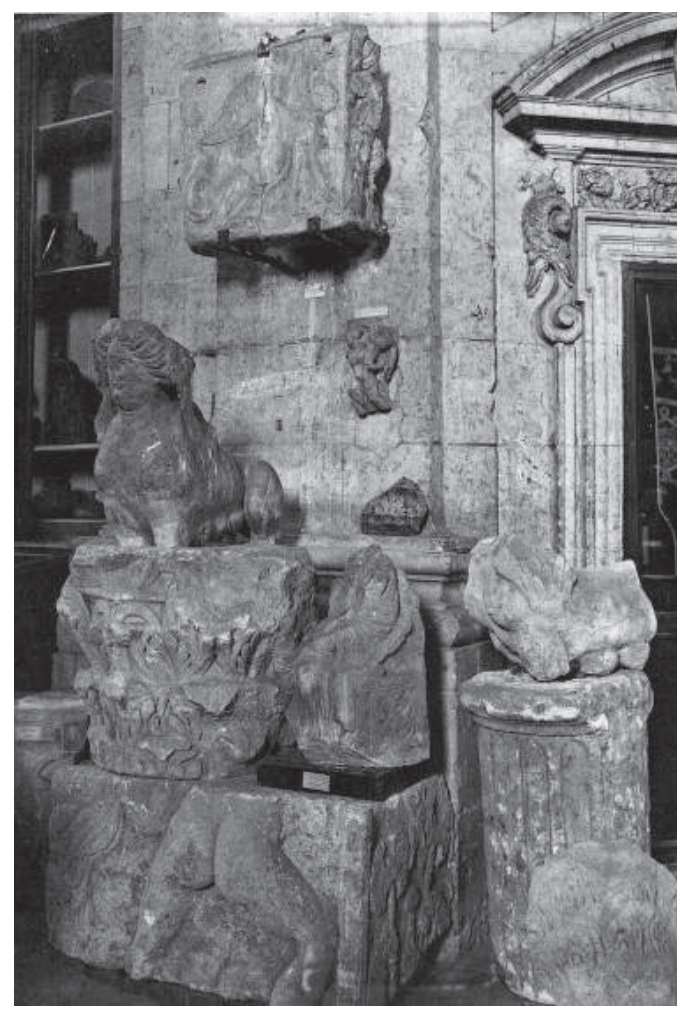

attendre 1893 pour voir publié, par Léon Maître, le premier véritable plan archéologique de la ville avec la représentation du tracé de l'enceinte tardive $^{67}$ (fig. 8) et le début des années 1980 pour que soient mises en œuvre les premières fouilles de sauvetage ${ }^{68}$. Aujourd'hui encore, en ce début de troisième millénaire, Nantes fait partie des villes d'origine ancienne parmi les moins bien connues de France et la réalisation d'une synthèse sur son histoire urbaine depuis l'Antiquité jusqu'au Moyen Âge est quasi impossible faute de données nouvelles issues d'une pratique raisonnée de l'archéologie préventive. Gageons que la création, en 2009, d'un service municipal d'archéologie permettra de pallier les importantes lacunes qui subsistent.

nantaise ", 303, 50, 1996, p. 4-19; SANQuER, René, Archéologie en Bretagne, 17, Spécial Nantes antique, 1978, 44 p.; Provost, Michel, CAG 44, La Loire-Atlantique, Paris, Académie des Inscriptions et Belles-Lettres, 1988, p. 81-106.

67. MAITTRE, Léon, Géographie historique et descriptive de la Loire-Inférieure, I, Les villes disparues des Namnètes, Nantes, Émile Grimaud, 1893, entre les pages 418 et 419.

68. Fouilles de l'hôtel de ville en 1980 et de l'école des Beaux-Arts en 1984, dirigées par Nicolas Rouzeau. 


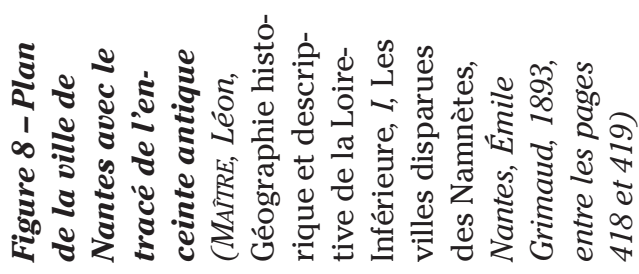

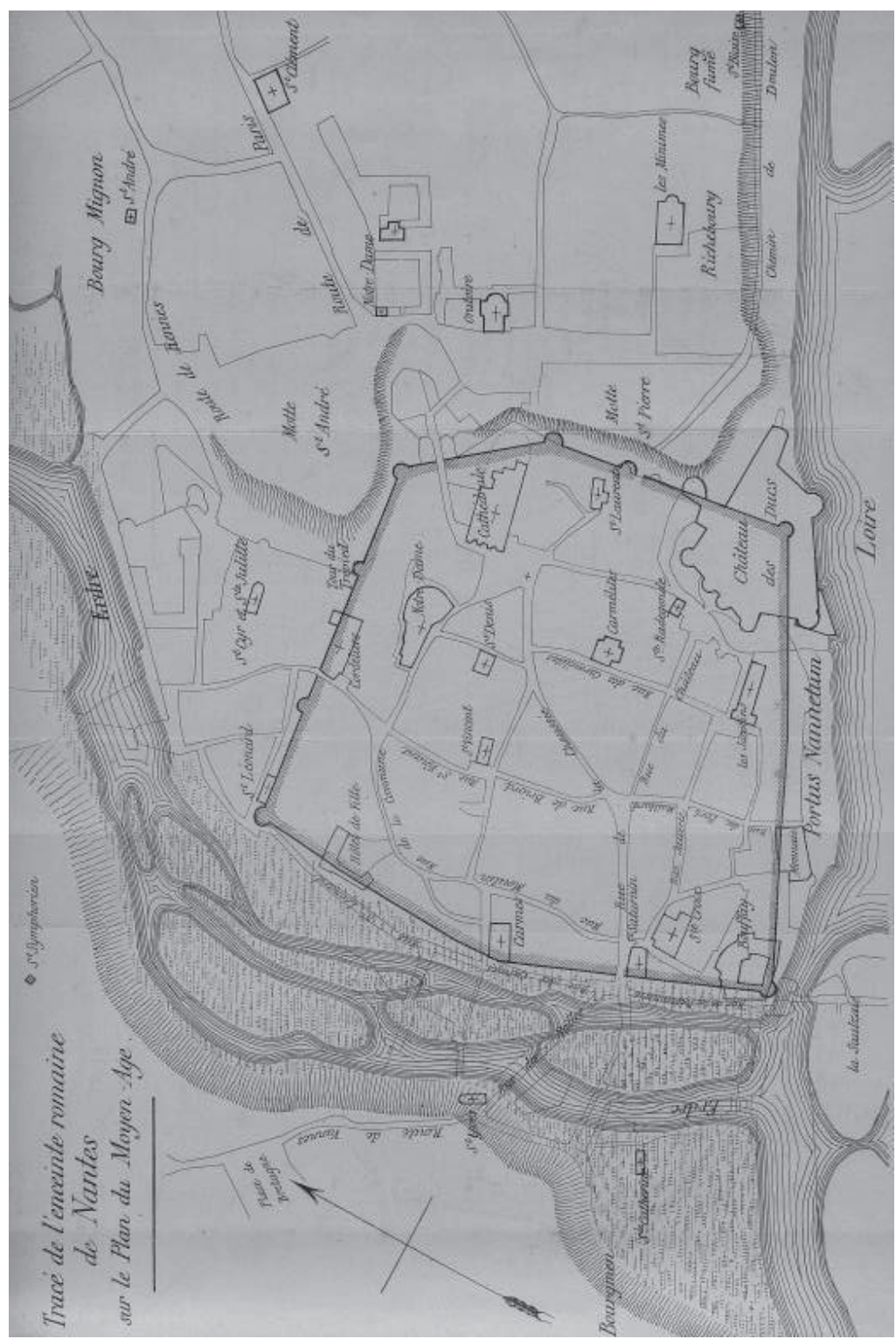




\section{RÉSUMÉ}

Les débuts de l'archéologie à Nantes, chef-lieu de cité antique et ville capitale à l'époque médiévale, sont lents, faute sans doute de monuments romains bien conservés en élévation et en raison de difficultés d'accès aux vestiges profondément enfouis dans le sous-sol. Exception faite de quelques découvertes fortuites, il faut en effet attendre la fin du XVIII ${ }^{\mathrm{e}}$ siècle et les observations pertinentes de Pierre-Nicolas Fournier pour que les premières données soient enregistrées avec méthode et surtout le milieu du XIX ${ }^{\mathrm{e}}$ siècle, à la suite de la création de la Société archéologique de Nantes et de la Loire-Inférieure, pour que les premières fouilles soient engagées et les objets recueillis exposés dans le cadre d'un musée.

\section{ABSTRACT}

The beginning of archaeology in Nantes, an administrative centre as an ancient city and a capital in the medieval period, was slow, undoubtedly for want of well-preserved Roman monuments above ground and the difficulties of access to remains buried deep underground. Except for some unexpected discoveries, it was not until the late $18^{\text {th }}$ Century and the pertinent observations of Pierre-Nicolas Fournier, that the first data were methodically recorded and especially not until the mid-1 $9^{\text {th }}$ Century, after the creation of the Société archéologique de Nantes et de la Loire-Inférieure, that the first excavations were undertaken and the objects found exhibited in a museum setting. 